\title{
Rapidly Growing Mycobacteria (RGM) And Pacemaker Infection
}

\section{Rayane Nassar, MD and Maha Assi, MD, MPH}

\section{Introduction}

- Infection rates of pacemakers and defibrillators range from $1 \%$ to $7 \%$.

- RGM known as M. fortuitum, M. abscessus, M.chelonae are uncommon pathogens of pacemaker infection.

\section{Case Presentation}

- A 68-year-old male with dilated cardiomyopathy $\mathrm{s} / \mathrm{p}$ defibrillator placement 3 years prior was admitted with a five-month history of fever, chills, and myalgias.

- Outside records:

- Blood culture positive for M. fortuitum, 5 months prior

- On admission:

- BP: 93/53 mmHg

- No focal signs of infection of generator pocket

- WBC: $4900 / \mu \mathrm{l}$

- $\mathrm{Hb}: 10.1 \mathrm{~g} / \mathrm{dl}$

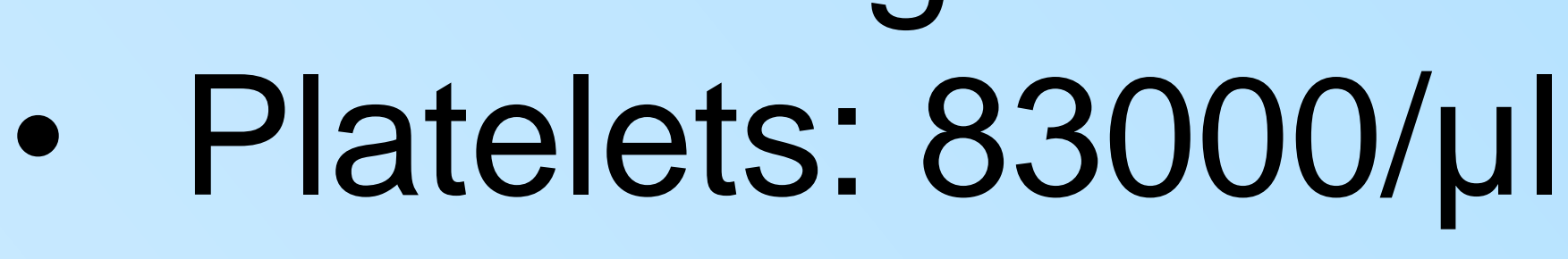

- Blood culture grew M. fortuitum

- Device infection was suspected.

- Defibrillator was removed and a temporary pacemaker placed.

- Defibrillator leads grew M. fortuitum.

- Therapy started with amikacin $10 \mathrm{mg} / \mathrm{kg}$ IV daily, cefoxitin 2 grams IV q 8 hours, and Levaquin $750 \mathrm{mg}$ IV daily.

- Blood culture was negative after one week of therapy.

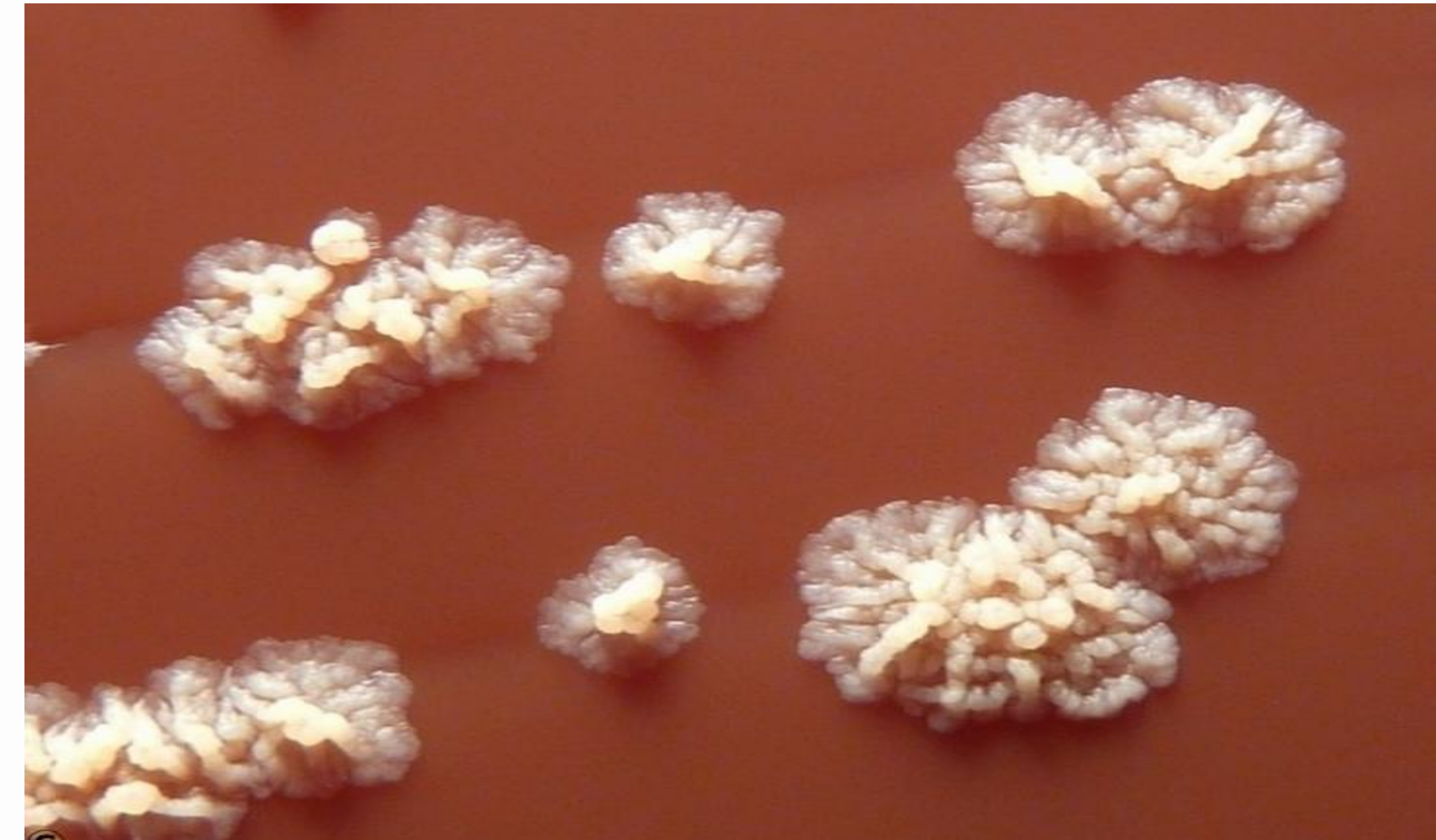

\section{Mycobacterium Fortuitum Blood Agar}

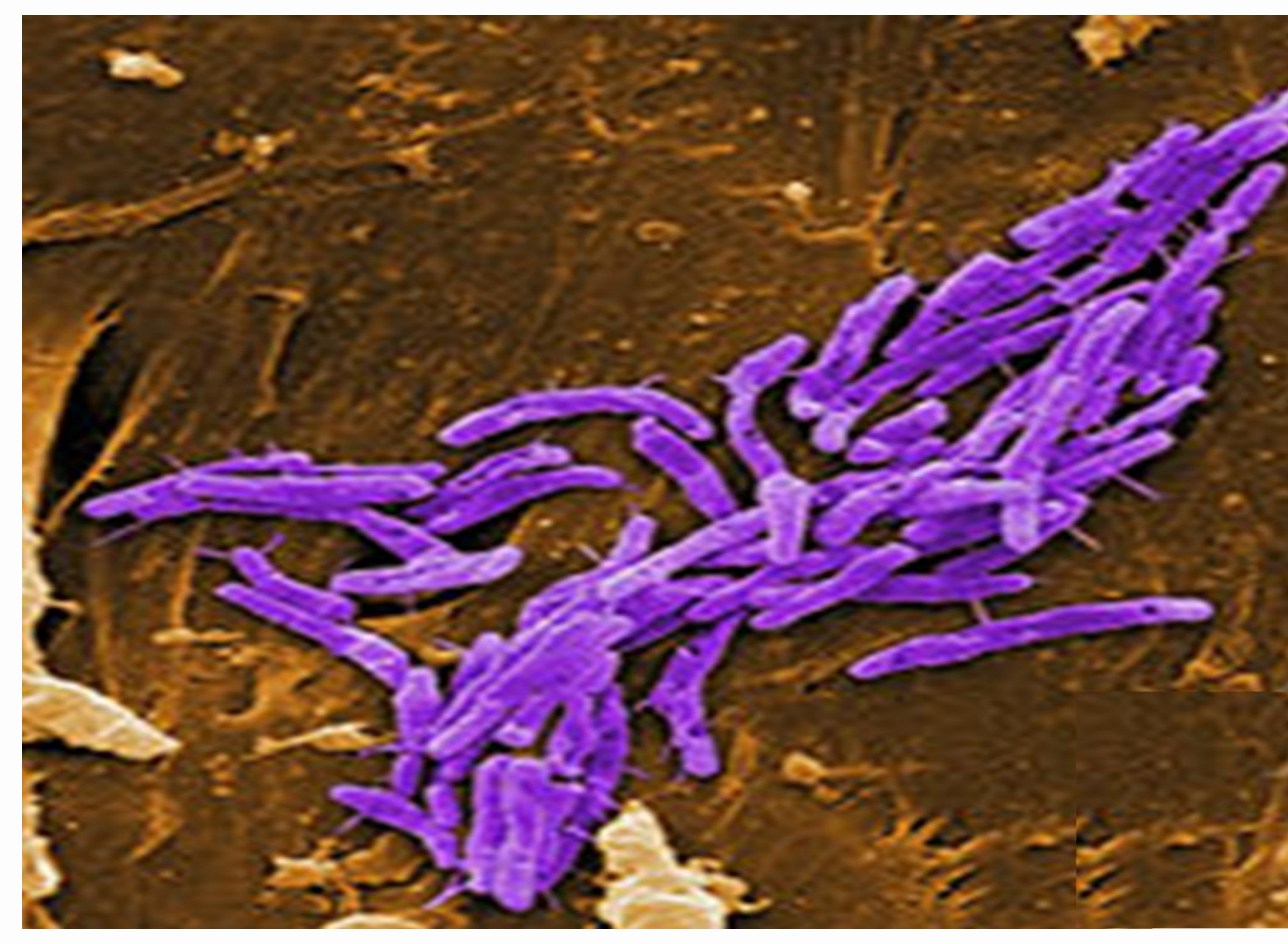

Mycobacterium Fortuitum

Scanning Electron Micrograph

\section{Discussion}

- RGM are ubiquitous in the environment and have an indolent disease course.

- It shows clinical signs after trauma or surgery.

- Disseminated disease is seen in immuno-compromised patients.

- Review of the English literature revealed 5 cases of RGM pacemaker infection.

- Infections are mainly nosocomial (within 6 months), but delayed onset was also reported ( $>1$ year).

- Suspect infection in patients with implanted cardiovascular devices even with absence of localized signs of infection on the generator site.

- Optimal treatment is removal of all device hardware, with empirical antibiotics until susceptibility results.

- RGM are in general susceptible to amikacin, fluoroquinolones, cefoxitin and linezolid

- The duration of therapy is 6 to 12 months.

\section{Take Home Messages}

$>$ Suspect device infection in patients with positive blood culture for RGM even in the absence of pocket inflammation.

Removal of hardware is crucial for curative therapy.

$>$ Start empiric IV antibiotics until susceptibility testing is available.

\section{References}

1. Wallace RJ, Brown BA, Griffin DE. Nosocomial outbreaks pseudo outbreaks caused by non tuberculous mycobacteria. Ann Rev Microbiol1998; $52: 453-490$.

2. Cutay AM, Horowitz HW, Pooley RW, Van Horn K, Wormser GP. Infection of epicardial pacemaker wires due to mycobacterium abscessus. Clin Infect Dis 1998; 26:520-521.

3. Kessler AT, Kourtis AP. Mycobacterium abscessus as a cause of pacemaker infection. Med Sci Monit 2004; 10:CS60-CS62.

4. Pastor E, Andreu AL, Llombart M. Mycobacterium Fortuitum: una rara causa de infeccion de marcapasos. Enferm Infecc Microbiol Clin 2006; 24:135-139. 